\title{
The dynamics of EEG correlates of activity of subcortical structures of the bird brain in conditions of chronic stress
}

\author{
Tatyana Pogrebnyak*, Elena Khorolskaya, Anzhelika Gorbacheva, and Irina Sagalaeva \\ Belgorod State National Research University, 308015, Belgorod, Russia
}

\begin{abstract}
In birds, acute stress (a three-day 12-hour inversion of the photomode) is associated with the dominant activity of the sympathetic hypothalamus and hippocampus, which suppress the tone of the parasympathetic section and the reticular formation of the midbrain. The effect of hyperglycemia on the background of a negative chronotropic effect indicates a mismatch of the functions of the autonomic sections of the hypothalamus on the 30th day. Chronic stress in birds (30-days crowding) causes persistent pathological stress of the functions of the anterior hypothalamus and reticular formation of the middle brain with the suppression of parasympathetic tone of the posterior hypothalamus and high functional activity of the hippocampus, determining the switching of the ventricles of the heart to a more economical mode of functioning by the 30th day. Using central cholinoblockers or tranquilizers, having a cholinoblocking component in the mechanism of its influence during neurogenic stress and in the post-stress period is excluded, but it is possible to use stressprotektors.
\end{abstract}

Keywords: chronic stress, EEG, subcortical structures.

\section{Introduction}

Stress refers to the manifestation of a neurogenic emotional state [1, 2]. However, in practice, its differentiation with this type of state is complex and can only be relative $[3,4]$. Physical stressors, as well as neurogenic ones, affect the emotional sphere, mental functions and nervous processes $[5,6]$. The development of a purposeful behavioral act with the manifestation of emotions against the background of neurogenic stress is associated with hypersynchronous electrical activity (EA) of subcortical structures of the brain [7, 8]. These emotional states have similar features: strengthening of protective and defensive reactions, inhibition of positive emotions and strengthening of negative ones with simultaneous violation of the vegetative functions of the body $[9,10]$.

When being stressed, especially in conditions of restricted motor activity, the limbic system and the reticular formation (RF) of the brain stem revealed the circulation of excitement [11], which contributes to the formation of a new neurotransmitter integration, triggering the entire complex of stress reactions in the body $[5,8]$.

\footnotetext{
* Corresponding author: pogrebnyak@bsu.edu.ru
} 
The activation of neurogenic stresses causes ordered $\theta$-activity in animals against the background of hypersynchronization of Hpt, RF and Hip, but desynchronous EA is registered in other parts of the brain at this moment $[2,12]$. The rhythmic EA of brain stem structures generates slow $\theta$ - frequencies that depend on the tone of the pituitary-adrenal system and ANS (autonomic nervous system) departments [13, 14]. In general adaptation syndrome, the amplification of $\theta$ - waves in subcortical centers is associated with the activity of the cortex and the sympathetic part of the posterior Hpt $[2,15]$. When the activity of both parts of the ANS increases, $\theta$-rhythm marks a period of maximum mobilization of internal resources of the body [3] against the background of relative dominance of sympathetic tone, which causes desynchronization of the EA structures of the brain stem regardless of stress $[5,13]$.

The correlates of the tone of nerve centers and mechanisms for regulating emotions are hypersynchronous and synchronized EA $[15,16]$. Against the background of hypoglycemia, hypersynchronized waves appear in the brain structures $[11,17]$. The activating influence of the limbic system causes the generation of $\theta$-rhythm in subcortical structures, and the activating effects of RF in the midbrain trigger desynchronization. The irritation of the caudate nucleus and anterior Hpt inhibits behavior and reduces RF tone in mammals [18, 19]. The inhibitory effect of anterior Hpt, Hip, and basal nuclei on the RF of the brainstem synchronizes its EA [5, 6].

The $\theta$-rhythm or stress rhythm is a specific biological correlate of emotional stress in biologically negative situations. [11]. This structure reflects the high activity of Hip in assessing the time signals of the environment, the genesis of emotions, and, obviously, in response to situational afferentation, it is able to synchronize its rhythm [1, 17].

Pathological EA of brain structures is reflected with a pronounced slow high-amplitude $\theta$-rhythm - rhythm, the generation of which is associated with the activity of the preoptic nucleus of the anterior Hpt when suppressing the functions of subcortical structures - RF and posterior Hpt [5, 15]. For example, the development of insulin hypoglycemia is associated with decreased energetic metabolism and the development of inhibition in the CNS (central nervous system) by suppressing functions of neurons, protecting them from depletion $[5,7]$. $\delta$-rhythm is associated with a braking behaviors, as it is most pronounced in the sleep state during the experiments in the development of internal inhibition [18]; correlation between inhibition of movements and development of $\delta$ - rhythm with the growth of the amplitudes of the individual low frequency and the reduction of the high ones under emergency cancellation of reinforcement of the conditioned stimulus [11, 18]. Hypersynchronuous activity corresponds to the level of development.

The aim of research is to study the dynamics of activity of subcortical structures of the brain of birds under chronic stress by the parameters of EEG correlates of stress, taking into account the dynamics of bird behavior, ECG indicators and the level of glycemia.

\section{Experiment}

The creating of a realistic model of chronic desynchronosis is described in a previously published paper [20]. Crowding, or densification of bird landing, is one of the most common stress factors in poultry farming, which causes hypoxia, a shift in the rhythms of daily components of stereotyped and motivated behavior, changes in the established hierarchical relationships in a group of birds, and deterioration of hygienic conditions [9, 21].

The preparation of birds for the experiment included: cupping the roosters ' crest, insertion of bipolar electrodes into the deep structures of the brain - anterior hypothalamus 
(Hpta), posterior hypothalamus (Hptp), reticular formation of the midbrain (RFmb) and hippocampus (Hip) using a stereotactic micromanipulator for rats [Fig. 1].

A

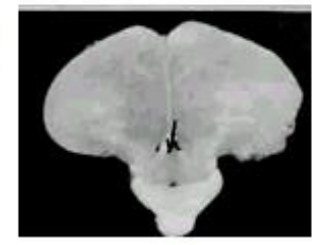

C

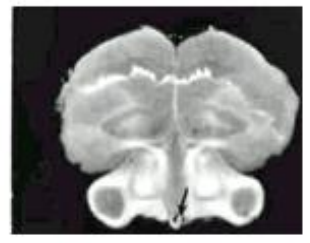

B

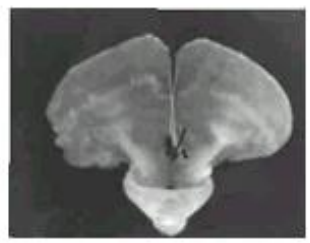

D

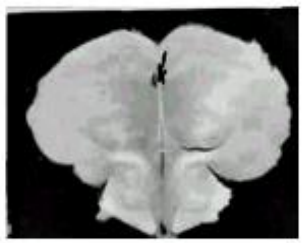

Fig. 1. Frontal section of the brain at the level of: A - Hpta, B - Hptp , C - Hip, D - RF midbrain; pointers- the location of the implanted electrodes. [20].

Coordinates of the immersion of electrodes in the brain structures of chickens (table 1).

A week after the stabilization of the functional state of birds according to the values of the level of glycemia and their behavior in a state of quiet wakefulness, the initial EEG was recorded bipolarly for 3-5 minutes (QW) and ECG in the second lead. The registration of EEG and ECG (Fig. 2) was performed 20 minutes after placing the birds into the machine in a soundproof chamber with 500 Lux illumination. During the registration of ECG and EEG, the roosters were in a specially made machine.

Table 1. Coordinates of immersion of electrodes into the structure of the brain of chickens [20].

\begin{tabular}{|l|l|l|l|l|}
\hline \multirow{2}{*}{$\begin{array}{l}\text { Distance from the tip } \\
\text { of the electrode to the } \\
\text { main plane }\end{array}$} & \multicolumn{4}{|c|}{$\begin{array}{c}\text { Implantation area of the } \\
\text { electrodes }\end{array}$} \\
\cline { 2 - 5 } & $\mathbf{H p t}_{\mathbf{n}}$ & $\mathbf{H p t}_{3}$, & Hip & RF \\
\hline $\begin{array}{l}\text { Sagittal, count from the } \\
\text { zero plane (F), mm }\end{array}$ & $-18,0$ & $-16,0$ & $-13,0$ & $-11,0$ \\
\hline $\begin{array}{l}\text { Front, count from } \\
\text { sagittal seam (L), mm }\end{array}$ & 1,0 & 1,0 & 1,0 & 1,0 \\
\hline $\begin{array}{l}\text { The depth of the } \\
\text { electrode, } \\
\text { counting from ground } \\
\text { plane (H) mm }\end{array}$ & $-9,5$ & $-8,0$ & $-3,0$ & $-12,5-$ \\
$-10,0$ & $-8,5$ & $-3,5$ & 13,0 \\
\hline
\end{tabular}

The model of desynchronization-crowding was created by increasing the density of placing five birds into single cages-up to $270 \mathrm{sm}^{2}$ per head. The birds could not move freely at such a density of landing; they either stood or sat. EEG and ECG were recorded before the experiment and then for a month on the 1st, 3rd, 7th, 14th, 23rd and 30th days (table 2).

The interpretation of the obtained information EEG indicators of the EA structures of the brain was carried out taking into account the description and analysis of its parameters in vertebrates-reptiles, birds and mammals $[11,13,19,20]$.

The desynchronization of EA included amplification of fast $\alpha$ - like activity and suppression of slower $\theta$ - and $\delta$ - ranges with a decrease in the amplitudes of all vibrations $[11,16]$. The suppression of fast $\alpha$ - like activity and amplification of slow $\theta$ - and $\delta$ - bands with an increase in the amplitude of their waves corresponded to synchronizing processes 
$[11,14]$. However, the increase in the severity of EA due to the growth of the wave amplitude simultaneously with a decrease in the overall frequency was estimated as a hypersynchronization reaction $[5,12]$.

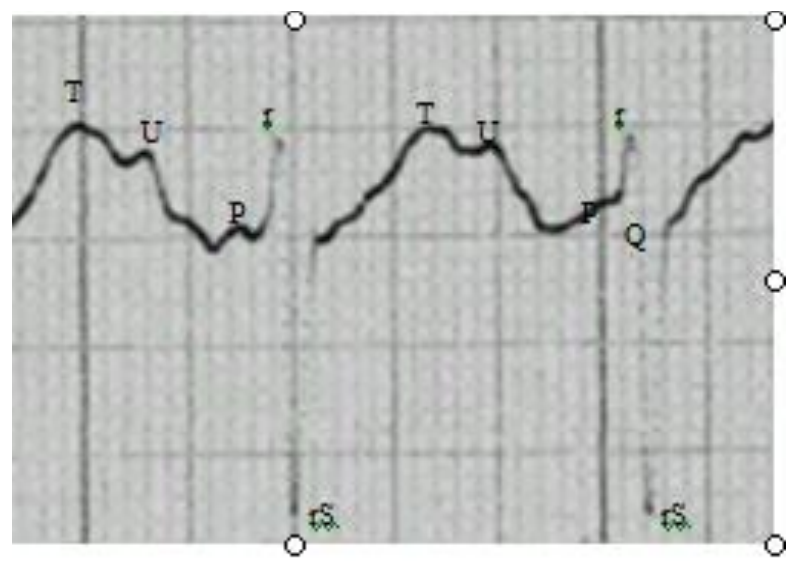

Fig. 2. Parameters of the cardiocycle: teeth P, Q, rS, T, U (II lead) [20].

Table 2. Model of chronic stress.

\begin{tabular}{|c|c|c|c|c|c|}
\hline \multicolumn{6}{|c|}{ Model of crowding } \\
\hline \multirow{2}{*}{ Stages } & \multirow{2}{*}{$\begin{array}{l}\text { № } \\
\text { Birds }\end{array}$} & \multicolumn{2}{|c|}{ Estimation } & \multicolumn{2}{|c|}{ Analysis } \\
\hline & & Behavior & Habit & ECG & EEG \\
\hline $\begin{array}{l}\text { Intact group } \\
\text { (control); }\end{array}$ & 10 & + & + & + & - \\
\hline $\begin{array}{l}\text { Experimental } \\
\text { group: }\end{array}$ & 10 & + & + & + & - \\
\hline $\begin{array}{l}\text { Before crowding } \\
\text { (background); }\end{array}$ & 10 & + & + & + & + \\
\hline $\begin{array}{c}\text { on days } 1,3,7,15 \text {, } \\
23 \text { and } 30 \text { of stress } \\
\text { (experiment) }\end{array}$ & 10 & + & + & + & + \\
\hline
\end{tabular}

The pair correlation analysis was used to consider the functional status of roosters as a manifestation of the process in all its variety of quantitative and qualitative indicators and their characteristics, to identify regulatory relationships between markers of stress and adaptation (glycemic level, ECG and EEG indicators) to determine the direction of shifts in the functional state of brain structures in the conditions of desynchronosis $[6,21]$. The correlation of EA shifts of the studied CNS structures with the level of glycemia was evaluated as a reflection of their central connections implemented through the peripheral vegetative link of regulation of visceral and somatic functions of the body [4, 15].d Determining its adaptability to the environment. To interpret the data obtained, we used the coefficients of paired correlations with their severity at least $0.66(\mathrm{p} .05 ; \mathrm{n}=10)$ and 0.71 $(\mathrm{p}<0.05 ; \mathrm{n}=8)$.

\section{Results and discussion}


The level of glycemia in intact and experimental birds before stress was observed within the normal range, amounting to 7.8-9.0 $\mathrm{mmol} / \mathrm{l}$ and 7.8-8.9 $\mathrm{mmol} / \mathrm{l}$, respectively. In intact birds, the average level was from 8.4 to $8.6 \mathrm{mmol} / \mathrm{l}$. In experimental birds, glycemia decreased on the 1 st day of crowding $(p<0,1)$ against the background and more significantly $(p<0,05)$ against intact birds, but on the 3rd day it recovered within the initial value. On the 7 th and 15th days, glycemia exceeded the background by $34.4(\mathrm{p}<0.05)$ and $30.9 \%(\mathrm{p}<0.05)$, respectively, and was higher $(\mathrm{p}<0.05)$ compared to intact ones. In the subsequent period- on the 23rd and 30th days, it decreased to the initial value.

In physiological conditions, the mixed Hpta EA of birds is marked by subdominating fast $\alpha$-waves with an amplitude of $15-35 \mathrm{mV}$, and slow $\theta$-waves with an amplitude of $30-40 \mathrm{mV}$. Crowding caused hypersynchronization of EA in Hpta - growth of $\delta$-waves on the 1st day, $\theta$-waves against the background by $26.1 \%(\mathrm{p}<0.05)$ on the 3 rd day, an increase in flattened $\delta$-waves. The reduction of $\alpha$-waves with the growth of their amplitudes in Hpta indicated high energy consumption and stress associated with hypoxia. The increase in Hpta $\theta$-rhythm $(-0.66)$ on the 3rd day of the experiment. Desynchronization of EA in Hpta on day 7 was accompanied by an increase in the amplitudes of $\theta$-waves by $46.5 \%(\mathrm{p}<0.01)$ above the background and a high expression of $\delta$-waves. On day 15 in Hpta, the hypersynchronization reaction was marked by an increase against the background of the $\delta$-factor. A large percentage of slow $\theta$-waves in the Hpta EA on day 1-15 indicated a high voltage of its functions with pathological brain activity against the background of inhibition of the functions of the ascending systems-RFSM and Hptp. The desynchronization of EA in Hpta on the 23rd and 30th days was accompanied by an increase against the background of the amplitudes of $\alpha$ - and $\theta$ - waves by $67.7(\mathrm{p}<0.01)$ and $57.1 \%(\mathrm{p}<0.01)$, respectively, with the manifestation of $\delta$-activity within the background. But on the 30th day there was a reduction in the $\theta$-rhythm by $29.0 \%$ ( $\mathrm{p}<0.05)$. A decrease in the level of glycemia $(+0.82)$ on day 23 directly correlated with a decrease in the total PE, and on day 30 with the $\alpha$-rhythm $(-0.66)$ and the amplitude of $\alpha$-waves (+0.67). These correlation pairs were marked, the regulatory relationship between the activation of the Hpta and decreased glycemic levels.

Hpt3. In physiological conditions, the Hptp EA of birds is represented by subdominating $\alpha$-waves $(20-35 \mathrm{mV})$, slow $\theta$-waves $(40-70 \mathrm{mV}$ ) with the manifestation of single slow $\delta$ waves with an amplitude of 45-70 $\mathrm{mV}$ against their background.

In Hptp, a desynchronizing process was observed with a flattening of $\theta$-waves by $26.4 \%$ $(\mathrm{p}<0.05)$, and a reduction of $\delta$-factor on the 1 st day of crowding. This EA was amplified on the $3 \mathrm{~d}$ day by reducing the amplitude of the $\theta$-waves by $28.1 \%(\mathrm{p}<0.05)$ and increasing the flattened $\delta$-waves. A decrease in the level of glycemia on the $3 \mathrm{~d}$ day was negatively correlated with an increase in the amplitudes of $\theta$-waves (-0.69). On the 7 th day, the activation reaction in Hptp increased - the total PE increased by $18.8 \%(p<0.05)$, the percentage of $\delta$-waves decreased to the background. On the 15th day, the Hptp synchronized EA showed an increase in the $\delta$-factor, which exceeded the initial value on the $23 \mathrm{~d}$ day against the background of desynchronization of the EA associated with an increase in the $\alpha$ rhythm by $14.4 \%(\mathrm{p}<0.05)$ and a decrease in the $\theta$-waves by $33.3 \%(\mathrm{p}<0.05)$; on 30th day, the desynchronizing effect was less pronounced.

The initial wave structure of the Hip EA is represented by dominant fast $\alpha$-waves with an amplitude of 20-35 mV, with slow 30-50 mV $\theta$-waves with an amplitude of and single $\theta$ waves with an amplitude of 35 to $75 \mathrm{mV}$. Crowding caused a hypersynchronization reaction in Hip with an increase in the number of $\delta$-waves on day 1 . On the $3 \mathrm{rd}$ and 7 th days in Hip increased $(\mathrm{p}<0.05) \alpha$ - and $\theta$-waves against the background of desynchronizing frequencies: on the 3rd day-reduction of $\theta$-rhythm by $27.2 \%(\mathrm{p}<0.05)$ and increase of $\delta$-waves; on the 7 th day, the total frequency increased by $13.6 \%(\mathrm{p}<0.05)$. On day 3 , the level of glycemia 
correlated with an increase $\alpha$-rhythm (+0.70) with flattening of the amplitudes of $\theta$-waves (0.68). These correlations indicated a regulatory relationship between Hip and the level of energy supply of the body. The increase in hypersynchronization in Hip on the 15th and 23rd days was marked by an increase $(\mathrm{p}<0.05)$ in the amplitudes of $\alpha$ - and $\theta$-waves; the percentage of $\theta$-waves increased on the 15 th day by $48.5 \%(\mathrm{p}<0.05)$ with a decrease in the $\delta$ factor, but on the $23 \mathrm{rd}$ day the percentage of $\delta$-waves increased. On the 30 th day, against the background of desynchronized EA, Hip showed unreliably high amplitude of $\alpha-$ and $\theta$ waves.

RFmb. In physiological conditions, the mixed EA RFmb of birds in the QW state was marked by the dominant fast $\alpha$-activity with an amplitude of $15-25 \mathrm{mV}$ and slower $\theta$-waves with an amplitude of $30-45 \mathrm{mV}$, single high-amplitude $\delta$ - waves. The background level of glycemia was inversely correlated with the total PE $(-0.76)$ RFmb of birds - its synchronizing activity.

The crowding caused the reaction of desynchronization in RF birds from the 1 st day to the 7 th. Its manifestation on the 1st day against the background was marked by a decrease in the $\delta$-factor. On the $3 \mathrm{~d}$ day, the RF showed desynchronizing processes, which were preserved on the 7 th day, and on the 15 th day the hypersynchronization reaction was preserved. During this period, hyperglycemia directly correlated with the growth of the $\alpha$ index $(+0.75) \mathrm{RF}$, noting the high stress of its functions. On the $23 \mathrm{~d}$ and 30 th days, the RFmb EA was desynchronized. Its manifestation on the $23 \mathrm{~d}$ day was marked by an increase in the $\alpha$-index by $13.1 \%(\mathrm{p}<0.05)$ and the amplitudes of $\alpha$ - and $\theta$-waves by $37.2(\mathrm{p}<0.05)$ and $48.2 \%(\mathrm{p}<0.05)$, respectively, and a decrease in $\delta$-waves by 2.6 times against the background. The correlation of the level of glycemia with the increase in the total PE $(-0.74)$ of the RFmb on the 23rd day of the experiment indicated a decrease in its functional activity in the conditions of stabilization of energy supply. On the 30th day, the RFmb showed desynchronizing frequencies, against which the amplitude of $\theta$-waves exceeded the background by $43.9 \%(\mathrm{p}<0.05)$.

The main direction of the dynamics of EA in birds'hearts during their adaptation to the 30-day period of stress was revealed - a negative chronotropic effect.

Background heart rate was $337.5 \pm 7.4$ min- $^{-1}$. On days $1-3$ of stress, it decreased by $13.5 \%$ $(\mathrm{p}<0.01)$ and remained at this level on the 7 th and 15 th days. In the subsequent period, the heart rate decreased and on the 30th day was $255.4 \pm 29.1 \mathrm{~min}^{-1}$, that is, $24.3 \%(\mathrm{p}<0.01)$ below the background. Thus, from the 1 st to the 30th day, the duration of rS-rS cardiocycles increased from $0.197 \pm 0.008 \mathrm{~s}$ to $0.235 \pm 0.010 \mathrm{~s}(\mathrm{p}<0.05)$, and compared with the background of $0.178 \pm 0.004 \mathrm{~s}$. Its elongation was more significant $(\mathrm{p}<0.01)$.

The Q-T interval increased from 1st day of crowding. In the period from the $3 \mathrm{~d}$ to the 30th days, its average duration increased from $20.0 \%(\mathrm{p}<0.01)$ to $37.8 \%(\mathrm{p}<0.01)$ against a background of $0.133 \pm 0.003 \mathrm{~s}$. The duration of the interval P-Q and T-P increased unreliably by the 30th day.

The amplitude of the $\mathrm{P}$ teeth increased by $50.0 \%(\mathrm{p}<0.05)$ against the background on the 1 st day, but on the $3 \mathrm{~d}$ day it decreased to the background, on day 7-30 it significantly exceeded it $(\mathrm{p}<0.01)$. The maximum excess of the background value by $66.2 \%(\mathrm{p}<0.01)$ occurred on the $23 \mathrm{~d}$ day. Changes in the amplitude of the $\mathrm{rS}$ teeth were unreliable, but during the entire period of crowding its increase on 7-30 th days exceeded the backgroundby $14.5-15.9 \%$. The voltage of the $\mathrm{T}$ teeth on the $1 \mathrm{st}$ and 7 th days was shown within the background, but on the 3rd and 15-30th days it was reduced by an average of $17.1 \%$, indicating a lack of energy.

The correlations of the background level of glycemia with ECG parameters were found: with the $\mathrm{T}$-wave $(0.71 ; \mathrm{p}<0.05)$, with the heart rate $(0.45)$, the $\mathrm{Q}-\mathrm{T}$ interval $(0.44)$, and 
negative (-0.44) with the T-R interval. On the 1 st day of stress, glycemia correlated with heart rate $(-0.79 ; \mathrm{p}<0.05)$, with an interval of $\mathrm{rS}-\mathrm{rS}(0.73 ;(\mathrm{p}<0.05)$.

The level of glycemia was negatively correlated with the interval P-Q on the 3rd (-054; $\mathrm{p}<0.05)$ and $23 \mathrm{rd}(-0.55 ; \mathrm{p}<0.05)$ days, on the 30th $(0.64 ; \mathrm{p}<0.05)$ and with the amplitude of the $\mathrm{P}$ wave on the 15 th day $(-0.54 ; \mathrm{p}<0.05)$.

In the background conditions, a number of correlations of ECG parameters with the EA of brain structures were obtained. So, heart rate was associated with the inactivation of the Hip in PR $(-0,78 \mathrm{p}<0.05)$, the interval $\mathrm{P}-\mathrm{Q}$ - with $\theta$ - index HTC $(0,84 ; \mathrm{p}<0.05)$ and PR Hip $(0,66 ; \mathrm{p}<0.05)$; the interval Q-T - with $\theta$-index Hpta $(0,84 ; \mathrm{p}<0.05)$; the interval of $\mathrm{T}-\mathrm{R}-$ with $\alpha$-index RF $(-0,52)$; a tooth $\mathrm{R}$ - with $\theta$ - the Hpta $(0,77 ; \mathrm{p}<0.05)$ and Hip $(0,82 ; \mathrm{p}<0.05)$. The $\theta$-wave correlated with $\theta$-index Hpta $(0.68 ; \mathrm{p}<0.05)$ and inactivation of Hip by PR $(-$ $0.76 ; \mathrm{p}<0.05)$.

Crowding has changed the nature of neural mechanisms and regulatory connections. On the 1st day, a negative relationship was observed between the heart rate and the $\alpha$ - index PF $(-0.66 ; \mathrm{p}<0.05)$ and the T-P interval with the $\alpha$-index Hip $(-0.89 ; \mathrm{p}<0.05)$. On the 3rd day noted a negative relationship with heart rate $\alpha$-indexes of both divisions of the Hpt $(-0,62)$; between the interval Q-T and $\theta$-index Hpta and Hip (and of -0.76 to 0.83 ; p <0.05), SP Hptp $(-0,63), \alpha$-index Hptp (0.66); the shortening of the interval T-R correlated with $\theta$-index Hip $(-0,60)$ and $\alpha$-index $\operatorname{HSM}(0,70 ; p<0,05)$; flattening of $\theta$-waves was associated with the stress functions Hptp and $\theta$-index $(-0,73 ; \mathrm{p}<0.05)$.

On the 7th day of crowding, the duration of $\mathrm{p}-\mathrm{Q}$ intervals correlated with the $\alpha$-index of Hip $(0.67 ; \mathrm{p}<0.05)$ and both Hpt divisions $(-0.60 ;-0.66, \mathrm{p}<0.05)$. The T-P interval was paired with the $\alpha$-index $(0.73 ; \mathrm{p}<0.05) \mathrm{Hptp}$, and the voltage of the T teeth with the $\alpha$-index Hptp $(-0.80 ; \mathrm{p}<0.05)$. On day 15 , the nature of correlations was more pronounced. The lengthening of the $\mathrm{P}-\mathrm{Q}$ intervals was marked by activation of Hip $(0.86 ; \mathrm{p}<0.05)$ and $\mathrm{RF}$ $(0.72 ; \mathrm{p}<0.05)$, and the T-P interval correlated with $\alpha$-index $(0.73 ; \mathrm{p}<0.05)$ and $\theta$-index $(0.92 ; \mathrm{p}<0.05)$ Hpta. An increase in the $\mathrm{P}$ tooth was associated with RF inactivation $(-0.73$; $\mathrm{p}<0.05)$, flattening of T-teeth with Hip inactivation by the $\alpha$ - index $(-0.72 ; \mathrm{p}<0.05)$.

In conditions of crowding on the 23rd day, the decrease in heart rate correlated with Hpta $(-0.89 ; \mathrm{p}<0.05)$ and RF $(-0.72 ; \mathrm{p}<0.05)$ and its $\theta$-index $(-0.62)$. The lengthening of the $\mathrm{p}-\mathrm{Q}$ intervals is associated with RF PE $(0.85 ; \mathrm{p}<0.05)$. The $\mathrm{Q}-\mathrm{T}$ interval correlated with an increase in Hpta PE $(0.71 ; \mathrm{p}<0.05)$. During this period, the growth of $\mathrm{P}$ teeth was associated with the RF-index RF $(-0.73 ; \mathrm{p}<0.05)$.

On day 30, the heart rate deceleration was associated with Hpta inactivation - with a decrease in the $\alpha$-index $(0.67 ; \mathrm{p}<0.05)$. Lengthening of intervals P-Q-with activation by PE Hptp (0.85; $\mathrm{p}<0.05)$ and $\alpha$-index RF $(0.69 ; \mathrm{p}<0.05)$, inactivation by $\theta$-index Hip (-0.71). For 30 days the lengthening of $\mathrm{Q}-\mathrm{T}$ intervals correlated with a decrease in the $\theta$-Hptp index. The growth of $\mathrm{P}$ teeth was associated with an increase in the $\alpha$-index Hpta $(0.81 ; \mathrm{p}<0.05)$ and activation of the PE Hptp $(0.66 ; \mathrm{p}<0.05)$ and Hip $(0.77 ; \mathrm{p}<0.05)$ and RF for PE $(0.89$; $\mathrm{p}<0.05)$; flattening of the T teeth - with an increase in the $\alpha$-index Hip $(0.68 ; \mathrm{p}<0.05)$.

\section{Conclusion}

The analysis of the revealed correlations allows us to note the determining value of the regulatory activity of Hpt departments, which is realized on the basis of activation of its vegetative departments and their synergy in the organization of adequate adaptation of the myocardial EA of the heart of experienced roosters to the conditions of chronic stress.

Crowding caused the birds on days 1-7 to increase the hypersynchronization reaction in Hpta by increasing the amplitudes of $\alpha$ - and $\theta$ - waves when in setting rarely the vibrations 
were reduced, especially in the first three days, and by increasing the flattened $\delta$-waves. Together, these changes indicated a high pathological stress associated with hypoxia. The manifestation of synchronization reaction in Hptp on the15th day of experiment is consistent with reduced functional voltage, and the manifestation of desynchronization reaction in it at 23-30 days notes its high functional activity.

The compaction of bird landing increased their reactivity and stress of Hptp functional activity during the entire period of crowding. Pathological Hpta stress from the 1st to the 15 th day was marked by a high level of $\delta$-activity, which is caused by neuro-emotional stress in the central nervous system and high tone of the parasympathetic Hpta department is associated with central inhibition. It was the increase in the General PE and $\alpha$-rhythm with the increase in the amplitudes of its waves on the 15th day of crowding that indicated the pathological stress of Hptp functions.

Thirty-days crowding of birds from the first day provided activation of birds Hip with its active participation in the formation of birds reaction to stress. Revealed on the $1 \mathrm{st}, 15 \mathrm{th}, 23 \mathrm{~d}$ days in Hip hypersynchronization EA was marked by increased $\theta$-rhythm, reflecting the emotional tension in the central nervous system and the organization of roughly-search reflexes in conditions of uncertainty. The decrease in the number of $\alpha$-waves with increasing their amplitudes in the Hip at 1-st, 15th, 23d days showed high voltage of its functions, perhaps related to hypoxia, and amid increasing of $\theta$-rhythm noted a mismatch in time of conditionally-reflex connections.

The crowding of birds caused an increase in desynchronizing processes in RF with the appearance of hypersynchronous waves of all ranges against their background. The manifestation of hypersynchronization reaction in it on the15th day marked high emotional tension of birds and deep neurogenic disorders in the central nervous system.

A sharp restriction of the flow of sensory afferentation as the main system-forming factor of functional systems in conditions of crowding caused the manifestation of hypersynchronous waves of all amplitudes in RF of birds. In addition, their manifestation in RF indicated its high pathological tension, associated with the manifestation of negative emotions, defensive and aggressive reactions caused by violation of the rhythms of the vegetative functions of the body.

\section{References}

1. F. Meerson, Successes fiziol. Sciences, 18, 4 (1987)

2. N. D. Ozernyuk, S. K. Nechaev, Izv. AN, ser. Biological, 4, (2002)

3. F. I. Furduy, V. P. Fedoryak, S. H. Haidarliu [at al.], Physiological mechanisms of stress and adaptation during acute exposure to stress factors (Science, Chisinau, 1986)

4. A. A. Filaretov, T. T. Podviga, L. P. Filaretova, Adaptation as a function of the pituitary-adrenocortical system (Science, SPb., 1994)

5. F. P. Vedyaev, T. M Vorobeva, Models and mechanisms of emotional stress (Health, Kiev, 1983)

6. B. M. Fedorov, Stress and circulatory system (Medicine, Moscow, 1991)

7. M. G. Pshennikova, The phenomenon of stress. Emotional stress and its role in pathology. Actual problems in pathophysiology (Medicine, Moscow, 2001)

8. F. I. Furduy, V. P. Fedoryak, S. H. Haidarliu [at al.], Physiological mechanisms of stress and adaptation during acute exposure to stress factors (Science, Chisinau, 1986)

9. Yu. P. Kvitkin., N. G. Federchenko and I. L. Krivtsov, Stress of poultry (Overview information, Moscow, 1977) 
10. A. I. Volozhin, Yu. K. Subbotin, Illness and health: two sides of adaptation (Medical, Moscow, 1998)

11. M. G. Amigarova, M. I. Arkhangelskaya, Successes are modern biology, 102, 1 (1986)

12. O. G.Baklavadzhyan, N. L.Pogosyan and A. V. Ashmyakan, Journal of Evolution. Biochemistry and Physiology, 26, 2 (1990)

13. V. M. Smirnov, Bulletin of Experimental Biology and Medicine, 130, 10 (2000)

14. A. A. Viru, Achievements of Physiological Sciences, 11, 4 (1980)

15. T. A. Pogrebnyak, G. I. Gorshkov, Scientific result. Physiology, 1 (2014)

16. M. M. Khananashvili, Pathology of higher nervous activity (Medicine, Moscow, 1983)

17. L. Kh. Garkavi, Ye. B. Kvakina and M. A. Ukolova, Adaptation reactions and resistance (Rostov University Press, Rostov, 1990)

18. S. Kh. Haydarliu, Mediator mechanisms of stress. Mechanisms of stress development (Science, Chisinau, 1987)

19. G. Horn, Memory, imprinting and the brain: a study of the mechanisms (World, Moscow, 1988)

20. T. A. Pogrebnyak, Scientific result. Physiology, 2, 3 (2006)

21. V. V. Matyushnikov, Natural resistance of poultry (Rosselkhozizdat, Moscow, 1985) 